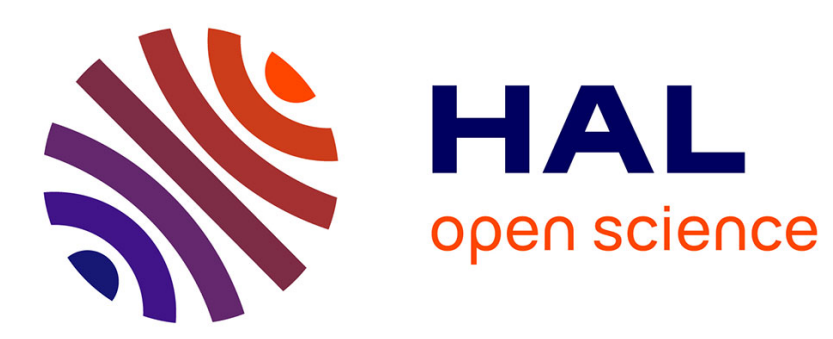

\title{
Une guerre sans héros? La guerre civile dans la bande dessinée espagnole (1977-2009)
}

Benoit Mitaine

\section{To cite this version:}

Benoit Mitaine. Une guerre sans héros? La guerre civile dans la bande dessinée espagnole (1977-2009). Cahiers de la Méditerranée, 2011, Guerres et guerriers dans l'iconographie et les arts plastiques, 83, pp.227-236. hal-01104394

\section{HAL Id: hal-01104394 \\ https://u-bourgogne.hal.science/hal-01104394}

Submitted on 21 Jan 2015

HAL is a multi-disciplinary open access archive for the deposit and dissemination of scientific research documents, whether they are published or not. The documents may come from teaching and research institutions in France or abroad, or from public or private research centers.
L'archive ouverte pluridisciplinaire HAL, est destinée au dépôt et à la diffusion de documents scientifiques de niveau recherche, publiés ou non, émanant des établissements d'enseignement et de recherche français ou étrangers, des laboratoires publics ou privés. 


\title{
Cahiers de la Méditerranée
}

83 (2011)

Guerres et guerriers dans l'iconographie et les arts plastiques

Benoît Mitaine

\section{Une guerre sans héros ? La Guerre civile dans la bande dessinée espagnole (1977-2009)}

\begin{abstract}
Avertissement
Le contenu de ce site relève de la législation française sur la propriété intellectuelle et est la propriété exclusive de l'éditeur.

Les œuvres figurant sur ce site peuvent être consultées et reproduites sur un support papier ou numérique sous réserve qu'elles soient strictement réservées à un usage soit personnel, soit scientifique ou pédagogique excluant toute exploitation commerciale. La reproduction devra obligatoirement mentionner l'éditeur, le nom de la revue, l'auteur et la référence du document.

Toute autre reproduction est interdite sauf accord préalable de l'éditeur, en dehors des cas prévus par la législation en vigueur en France.
\end{abstract}

\section{revues.org}

Revues.org est un portail de revues en sciences humaines et sociales développé par le Cléo, Centre pour l'édition électronique ouverte (CNRS, EHESS, UP, UAPV).

Référence électronique

Benoît Mitaine, "Une guerre sans héros ? La Guerre civile dans la bande dessinée espagnole (1977-2009) »,

Cahiers de la Méditerranée [En ligne], 83 | 2011, mis en ligne le 15 juin 2012, consulté le 10 janvier 2015. URL:

http://cdlm.revues.org/6256

Éditeur : Centre de la Méditerranée moderne et contemporaine

http://cdlm.revues.org

http://www.revues.org

Document accessible en ligne sur :

http://cdlm.revues.org/6256

Document généré automatiquement le 10 janvier 2015. La pagination ne correspond pas à la pagination de l'édition papier.

(c) Tous droits réservés 


\author{
Benoît Mitaine
}

\title{
Une guerre sans héros ? La Guerre civile dans la bande dessinée espagnole (1977-2009)
}

\author{
Pagination de l'édition papier : p. 227-236
}

Il devient de plus en plus difficile de parler de la Guerre civile espagnole sans auparavant signaler qu'après la mort du général Franco, en 1975, une autre guerre a pris le relais, centrée sur l'intarissable question de la mémoire historique. Il s'agit d'une guerre d'historiens, de journalistes, d'intellectuels, d'artistes, de juristes, de politiques et de citoyens militants qui se livrent une guerre de chiffres et d'idées avec pour objet central la volonté, pour les uns, de réparer une partie des crimes et des injustices perpétrés par les vainqueurs sur les vaincus et, pour les autres, de réhabiliter la mémoire de Franco et l'œuvre du franquisme.

L'engouement du public pour ce sujet est sans cesse relancé par des polémiques, des débats parlementaires et des lois, des commémorations, des expositions, des événements éditoriaux (romans ou essais), des reportages ou des films qui avivent en permanence le souvenir encore vif d'une guerre à présent vieille de 70 ans (1939-2009). D'un point de vue éditorial, le sujet est devenu incontrôlable au point d'avoir échappé depuis des années à toute comptabilité ${ }^{1}$. Et pourtant, au sein de ce panorama socioculturel fiévreux et chaque jour plus proche, semble-til, de la saturation, il existe un secteur éditorial qui est passé à côté de ce formidable filon doré qui a fait le bonheur de tant d'éditeurs. Je veux ici parler de la bande dessinée.

\section{Une guerre contre soi-même}

Nous sommes ici face à ce qui pourrait ressembler à un paradoxe. D'une part, le secteur de la bande dessinée n'est pas réputé pour sa frilosité devant l'argent facile et les bons coups et l'on pourrait donc déjà se demander pourquoi le filon de la mémoire historique est resté sousexploité là où d'autres y ont fait leurs choux gras. D'autre part, s'il est bien un médium qui a su cultiver la figure du guerrier et du héros au point d'en faire une de ses marques de fabrique, c'est bien la bande dessinée :

Après la littérature d'aventures du XIX ${ }^{\mathrm{e}}$ siècle [...], la bande dessinée appartient aux dispositifs de fiction qui glorifient l'imaginaire multiforme de l'aventure comme récit héroïsé du dépassement de soi dans la quête courageuse du bien. Elle décline une «mystique moderne » liée à la figure romantique et individualiste de l'aventurier en tous ses états, parfois militaire, souvent savant, explorateur, marin, globe-trotter ou sportif, $[\ldots]^{2}$.

Bien entendu, et c'est là où se dissout notre apparent paradoxe, toutes les guerres ne se valent pas. Les guerres anciennes ne sont pas les guerres modernes et les guerres nationales (fratricides) ne sont pas les guerres internationales.

Remarquons tout d'abord au sujet de la première dichotomie (ancien $v s$ moderne) qu'il existe une dynamique générale au niveau de l'histoire européenne qui tend vers un effacement progressif des figures guerrières de premier plan entre les $\mathrm{XV}^{\mathrm{e}}$ et $\mathrm{XIX}^{\mathrm{e}}$ siècles. C'est ce que montrent fort bien Jérôme Delaplanche et Axel Sanson dans Peindre la guerre où l'on comprend, par le texte et l'image, que la figure du guerrier s'estompe au fil des siècles pour finir par disparaître complètement au XIX ${ }^{\mathrm{e}}$ siècle avec l'industrialisation de la guerre : le temps de « l'holocauste » (le héros sacrifié) n'est plus et ne reste que «l'hécatombe » (la mort à l'échelle industrielle), autre façon de dire que nous sommes passés du guerrier à la guerre. L'extinction progressive du guerrier telle que l'a consignée la peinture au cours des siècles est d'ailleurs confirmée dans les dictionnaires contemporains pour qui le mot guerrier « ne s'emploie qu'en parlant des gens de guerre du passé, des civilisations préindustrielles ${ }^{4}$. Ces changements d'échelle (la massification des armées et la mondialisation des conflits) et de technique, en même temps qu'ils auront eu la peau du guerrier, auront aussi eu celle du 
peintre batailliste et du genre auquel il donnait vie. Dans les deux cas, la mécanisation et l'automatisation sont en cause : la gâchette du fusil et de la mitrailleuse rendront inopérants le sabre au même titre que la gâchette de l'appareil photo et de la caméra disqualifieront le pinceau.

Remarquons ensuite, au sujet de la seconde dichotomie (nationale $v$ s internationale), que l'on voit bien mal comment la Guerre civile espagnole, à la différence de la guerre d'Indépendance espagnole de 1808-1814 contre Napoléon ${ }^{5}$, pourrait servir de quelque façon à l'édification d'une geste nationale autour de laquelle l'ensemble du peuple pourrait se retrouver et se rassembler. Même si cette croisade contre le communisme a bien eu valeur de geste nationale pour certains et même si d'un point de vue historique il s'agit d'un événement fondateur ${ }^{6}$ de l'Espagne contemporaine, il ressort très clairement, aux yeux de l'Humanité, que dans cette guerre, ce sont les « méchants » qui ont gagné, si l'on veut bien utiliser un vocabulaire axiologique enfantin et facilement associable à l'univers manichéen de la bande dessinée. L'événement est certes fondateur mais difficilement unificateur. En outre, la guerre ne s'est pas arrêtée en 1939 une fois le conflit armé fini, mais, pour beaucoup d'Espagnols, en 1975, après trente-six ans de dictature. On comprendra donc aisément que dans ce contexte, la bande dessinée espagnole actuelle ait du mal à trouver des héros dignes d'admiration, pour autant d'ailleurs qu'elle en ait un jour cherchés?.

Ainsi, dans son acception la plus traditionnelle ou classique, la bande dessinée espagnole d'aventure et de héros ne semble pas avoir de prise sur cette matière historique, à la différence du roman. Toutefois, l'absence de héros et d'aventures associés à la guerre de 1936-1939 ne doit pas conduire à conclure ipso facto que ce sujet soit resté en Espagne à l'état de friche diégétique, car là où la bande dessinée commerciale a échoué jusqu'à présent, la bande dessinée dite d'auteur, indépendante et adulte s'est emparée du sujet avec un sérieux et un talent tels que de cette veine thématique sont nées quelques-unes des œuvres les plus remarquables de l'histoire de la bande dessinée espagnole ${ }^{8}$.

\section{Une production restreinte}

Pour être exact, et pour autant que mes recherches soient exhaustives, la production espagnole totale de ces trente-trois dernières années sur la Guerre civile et le franquisme, c'est-à-dire une période couvrant quarante années de l'histoire du XXe siècle espagnol (1936-1975), s'élèverait à 26 albums et 21 nouvelles graphiques.

On pourrait penser, à condition de placer derrière chaque titre un auteur différent, que ce corpus n'a rien de négligeable. Or, en regardant dans le détail cette bibliographie, un phénomène saute aux yeux : Carlos Giménez est l'auteur, à lui seul, de quatorze albums (Paracuellos : 6 vol. ; Barrios : 4 vol. ; 36-39. Malos tiempos : 4 vol.) ; Victor Mora est l'auteur de huit scénarios ; Antonio Hernández Palacios d'une tétralogie ; Felipe Hernández Cava de quatre scénarios ; Jorge García de trois scénarios. Soit un total de 33 titres sur 47 produits par seulement cinq auteurs. Quant aux maisons d'édition espagnoles qui s'intéressent à cette thématique, elles ne sont guère plus nombreuses et, hormis Glénat Espagne ${ }^{9}$, elles sont toutes de dimensions modestes et spécialisées dans l'édition de bandes dessinées d'auteur destinées à un lectorat adulte. Les Edicions de Ponent, de par leur implication éthique dans la constitution d'un patrimoine iconographique de nature bédéistique sur la mémoire des vaincus, illustrent bien cette tendance.

\section{Dessiner la guerre}

10 Au cours de ces trois dernières décennies, trois générations de dessinateurs se sont engagées sur ce terrain à haut risque de la représentation de la guerre : les aînés ont vu le jour au tout début des années 1920 (J. Blasco, A. H. Palacios), les cadets dans la seconde moitié des années 1970 (Fritz, J. García, F. Martínez) et la génération médiane, la plus importante, s'étend de 1941 à 1955 (C. Giménez, F.H. Cava, M.A. Gallardo, A. Altarriba, etc.). Bien qu' au sein de cet ensemble règne la diversité stylistique et thématique la plus totale, un parti pris graphique se dégage depuis le début : le rejet massif de la couleur. Le pouvoir de séduction de la couleur est tel qu'il existe toujours un risque à vouloir l'utiliser : soit qu'elle provoque l'admiration au lieu 
de la répulsion, soit qu'elle détourne l'attention sur le détail (le sang, la chair) au détriment de l'ensemble. L'hypothèse d'une monochromie imposée d'en haut par l'éditeur pour des raisons financières et non d'un choix graphique de l'auteur n'est jamais totalement à rejeter, mais comme elle ne saurait suffire à expliquer cette dominante, une conclusion s'impose : le noir, en tant que symbole de la douleur, du deuil et de la mort, est la couleur qui sied le mieux à la guerre.

\section{Antonio Hernández Palacios}

Cette doxa connaît, bien entendu, quelques auteurs qui dérogent à la règle. C'est notamment le cas d'Antonio H. Palacios, le doyen des auteurs espagnols et l'un des rares à avoir voulu coloriser une guerre que nous ne connaissons quasiment qu'en noir et blanc.

Dans cette œuvre, le choix de la couleur est cohérent avec l'approche thématique : là où les autres auteurs ont abordé la guerre sur le mode intimiste, critique ou autobiographique, ce spécialiste des fresques historiques s'est lancé dans une reconstitution minutieuse de la guerre (assauts, manœuvres, armements, costumes) sans vraiment porter de jugement (je m'en explique plus tard). Plus qu'une volonté de revenir sur le mode autobiographique sur ses propres années de guerre, le vétéran Palacios ${ }^{10}$ accepte de relever le pari que lui a lancé Ernesto Santolaya (directeur des éditions Ikusager) de dessiner la Guerre civile espagnole et part dans une aventure qui durera huit ans (1979-1987). Cette commande éditoriale fera de Palacios le premier et dernier (car unique) des dessinateurs bataillistes de la Guerre civile. Il est vrai que sa formation (les Beaux-Arts de Madrid) et son expérience (il avait déjà raconté la vie en bande dessinée du Cid, de Roland, de Christophe Colomb, de Philippe II et de Simon Bolivar) faisaient de lui l'un des dessinateurs les plus chevronnés pour ce genre de commande.

Loin de chercher à donner une vue d'ensemble du conflit, il préférera concentrer son attention sur diverses batailles : Toledo et l'Alcázar, la bataille de Madrid et le Pays Basque (Ikusager étant une maison basque, il est raisonnable de penser que ce choix relève plus de l'éditeur que de l'auteur). Tout au long de sa tétralogie, la population civile, l'arrière-front de même que toute forme d'individualisation des soldats (leur passé, leur vie, leur famille, leurs amours), seront en grande partie ignorés au strict bénéfice de la bataille et de l'action. En peintre fidèle des grandes épopées de l'Espagne, Palacios tend le plus possible à la neutralité et à l'objectivité ${ }^{11}$. Certes, ses personnages principaux sont toujours du côté républicain ${ }^{12}$, mais cela n'empêche pas Palacios de louer aussi bien la vaillance et la ténacité des troupes «rebelles » (qui, c'est à souligner, ne sont jamais appelées « fascistes ») que le courage et le sens du sacrifice des républicains. Dans cette optique descriptiviste où seul l'assaut a de l'importance, la quadrichromie sert efficacement le dessin / dessein de Palacios. Les couleurs lourdes et dégradées qui se répandent tout au long des quatre volumes reflètent bien l'âpreté des combats, l'épuisement des hommes et l'état de dégradation du pays à cette époque. Sous son crayon, l'Espagne entière semble plongée dans un immense halo de poussière, de poudre et de fumée où le chaos règne en maître. À cela se superpose une esthétique de la saturation des planches et des cases par un procédé d'accumulation (de couleurs, mais aussi d'explosions, de coups de feu, d'hommes, de mouvements) qui donne le vertige et qui ne fait que rajouter du trouble à cette immense mêlée fratricide. Les bulles, comme autant de trous dans le dessin, participent elles-mêmes de cette débauche en faisant écho graphiquement aux explosions et aux impacts (fig. 1). Enfin, l'agencement très sophistiqué des cases donne beaucoup de dynamisme à l'ensemble et sert efficacement une action toujours partagée en une multiplicité de lieux. On appréciera par exemple dans l'illustration ci-présente (fig. 2) comment le découpage vertical des cases en longues tranches étroites démultiplie la vitesse et la violence du coup de crosse asséné par le soldat franquiste (troupes marocaines) en plein assaut. Le mouvement qui fend l'air et les chairs dans la réalité, déchire l'espace paginal dans la fiction.

Bien que l'héroïsme et le courage ne fassent pas défaut dans cette tétralogie comme on peut le voir encore ici (fig. 2) avec ce soldat bravant la mort avec une agressivité et une agilité qualifiée de féline ( «saltan de casa en casa como gatos terribles »), aucune figure guerrière ne se détache de l'ensemble. Eloy ou Gorka, deux soldats que Palacios a véritablement connus durant la Guerre civile, ne sont que les participants d'un événement qui les dépasse et pour 
lequel ils ne témoignent d'aucune ardeur guerrière. Eloy (tout comme Gorka et les autres), comme le signale sans équivoque le sous-titre, n'est qu'un soldat parmi tant d'autres («Uno entre muchos »), formule qui fait d'emblée de lui un anti-héros.

\section{Carlos Giménez}

Carlos Giménez, né en 1941, soit vingt ans après Hernández Palacios, est peut-être bien, comme cela figure en quatrième de couverture de ses albums, « l'auteur le plus important de la bande dessinée espagnole de ces trente dernières années $»^{13}$. À la différence de Palacios, il n'a pas vécu la guerre, mais il en a enduré toutes les conséquences : orphelin de père et élevé avec ses deux frères par une mère valétudinaire qui souffrait de tuberculose, il sera placé à l'âge de six ans et jusqu'à ses quatorze ans (entre 1947 et 1955) dans les auxilios sociales, ces centres sociaux ayant aussi fonction d'orphelinat. Cette institution fondée en 1936 sur le modèle nazi des winterhilfe lui laissera un souvenir aussi noir qu'indélébile d'une après-guerre marquée par la privation (l'absence du père, la séparation avec la mère et le reste de la fratrie, la claustration, les pénuries alimentaires et autres) et par une éducation de fer fondée sur un autoritarisme militaro-religieux propre à la doctrine nationale-catholique des premières années du franquisme. Paracuellos, l'œuvre la plus connue et aussi la plus forte de Giménez, est le récit de cette expérience. Toutefois, ce n'est pas de cette dernière œuvre que nous allons parler ici, mais de sa tétralogie 36-39. Malos tiempos ${ }^{14}$, œuvre largement centrée sur la population civile durant le siège de Madrid par les troupes franquistes.

La grande majorité des cinquante-trois histoires qui composent les quatre albums de la série tourne autour de Marcelino, Lucía et de leurs quatre enfants : Paula, Sole, Carmen et Marcelino. À travers les six membres de cette famille ordinaire - le père ouvrier républicain et la mère au foyer -, Giménez va démultiplier les points de vue pour rendre compte de façon polyphonique du quotidien à la fois ordinaire et extraordinaire des habitants d'une ville assiégée et bombardée pendant près de trois ans. Ainsi, chacun des membres de la famille aura sa fonction narrative en agissant en tant qu' introducteur de milieu (social, professionnel) ou de sphère (publique, privée). Certes, les situations se répètent et les rôles se croisent sans cesse, mais c'est d'abord par le regard de Marcelino père, en tant qu' ouvrier et républicain, que l'auteur parvient, entre autres exemples, à dévoiler au lecteur l'ambiance révolutionnaire qui règne dans les rues et les usines aux premières heures du soulèvement (la panique du patronat ; les discussions politiques entre hommes dans les cafés ; les exécutions, etc.). Lucía, par son rôle de femme au foyer et de cuisinière, témoigne pour sa part des efforts surhumains qu'il faut accomplir en temps de guerre dans une ville assiégée pour nourrir une famille entière quand tout fait défaut et que la mort par malnutrition est devenue un fléau comparable aux bombardements. Cette comparaison macabre est d'ailleurs admirablement synthétisée dans les deux cases de l'illustration 3 ci-présente (vol. 3, p. 7). La quête et l'angoisse alimentaires sont telles qu'elles sont à l'origine de près d'un quart des récits, allant jusqu'à occuper la moitié des histoires du volume 2 de la série. Dans un récit intitulé «El pan de Franco » (t. 2, p. 28-30), on voit le peuple madrilène en train de brûler des pains largués au-dessus de la ville par l'aviation franquiste tout en scandant qu'il vaut mieux mourir affamé qu'humilié. Scène qui inspire à Lucía, modèle de pragmatisme et de tempérance, la formule suivante : «prefiero hijos sin honra, que honra sin hijos » (je préfère des enfants sans honneur, que l'honneur sans mes enfants).

17 Quant aux enfants, ceux de Marcelino et les autres, leur rôle actanciel est multiple : leur jeune âge fait d'abord d'eux les cibles privilégiées de la famine et des maladies (tuberculose en particulier). Ils apparaissent à ce titre comme les premières victimes indirectes de la guerre : la couverture du dernier album de la série (fig. 4) représente d'ailleurs un enfant alité qui, en voyant entrer dans sa chambre un prêtre flanqué de trois enfants de chœur, comprend brutalement qu'il va mourir. Pointant son doigt en direction du prêtre il s'exclame : « i...Que no me quiero morir... ! ; Que solo soy un niñooooo... ! » (Je ne veux pas mourir ! Je ne suis qu'un enfant !). Loin d'apporter un quelconque réconfort, cette cohorte macabre rappelant la Santa compañía incarne bel et bien la Mort sur cette image. En outre, et c'est le plus important, cette irruption violente des représentants du culte dans la sphère privée, irruption allant à l'encontre 
de la volonté et des croyances de la mère (qui est aussi atterrée que son fils), est le signe que la « croisade du bien contre le mal » est terminée tant sur le plan spirituel que militaire : c'est le triomphe des forces réactionnaires (fig. 4).

Ajoutons encore que les enfants, quand ils ne sont pas malades, sont bien plus actifs que passifs. Privés d'école, ils deviennent les parfaits auxiliaires des parents dans l'économie domestique : chargés de la collecte de bois pour alimenter le fourneau, ils glanent sur leur passage tout ce qui peut servir à améliorer le quotidien (cf. « Sole », t. 2, p. 9-12). Ils ne sont toutefois pas complètement désinfantilisés puisque le ludisme continue de faire partie intégrante de leur vie : leur terrain de jeu favori, en dépit des risques, reste très majoritairement la rue, ce qui fait d'eux les oreilles et les yeux de la sphère publique, les témoins directs des horreurs de la guerre (cf. «La bomba incendiaria », t. 2, p. 17-19).

Cette description de 36-39. Malos tiempos axée sur les personnages, aussi courte et schématique soit-elle, suffit amplement pour comprendre que le parti pris de Giménez est diamétralement opposé à celui de Palacios. Tout oppose ces deux tétralogies : le graphisme de l'un est baroque quand celui de l' autre (Giménez) est minimaliste, voire simpliste ; la débauche chromatique de Palacios, encore empreinte de l'esthétique hippie des années 1960, fait face à l'austère monochromatisme né de la vague underground des années 1970 ; les personnages de l'un se composent intégralement d'officiers et de soldats là où l'autre ne fait intervenir que des civils ; pour ce qui est de l'espace, le front cède la place à l'arrière-front et la sphère publique à la sphère privée, familiale ; les cadrages répondent aux mêmes antagonismes : les grands angles et les plans d'ensemble de Palacios s'opposent aux innombrables plans serrés mettant en scènes des dialogues ; enfin, l'arthrologie ${ }^{15}$ (i. e. l'enchainement des cases) complexe de Palacios est remplacée par un implacable gaufrier en six cases isomorphes chez Giménez. En conclusion, l'on observera qu'au milieu de cet océan de différences, les deux auteurs concordent dans le traitement de leurs personnages pour rejeter la figure du héros. Pour autant, et sans que cela soit contradictoire, l'un comme l'autre se sont attachés à héroïser une figure traditionnellement exclue des récits de guerre : le vaincu. Eloy, Gorka, mais aussi Lucía, cette femme au foyer qui réussit à faire manger quotidiennement six personnes, sont, chacun à leur façon, héroïques. Ce phénomène d'héroïsation des vaincus n'est pas propre à Hernández Palacios ou Giménez : il se retrouve aussi bien dans La guerra civil española, série de huit récits scénarisés par Víctor Mora et publiés dans la revue CIMOC entre 1986 et 1987, Un largo silencio (1997) de Miguel Ángel Gallardo, Cuerda de presas (2005) de Jorge García et Fidel Martínez, Nuestra guerra civil (œuvre collective, 2006) ou encore El arte de volar (2009) d'Antonio Altarriba et Kim.

L'engagement de la bande dessinée espagnole dans le long combat de la récupération de la mémoire historique des perdants reste timide comparé à d'autres arts, mais l'obtention récente des prix les plus prestigieux en matière d'arts graphiques à des œuvres portant sur la guerre civile $^{16}$ ne sera certainement pas sans conséquences sur la production des années à venir. 


\section{Annexe}

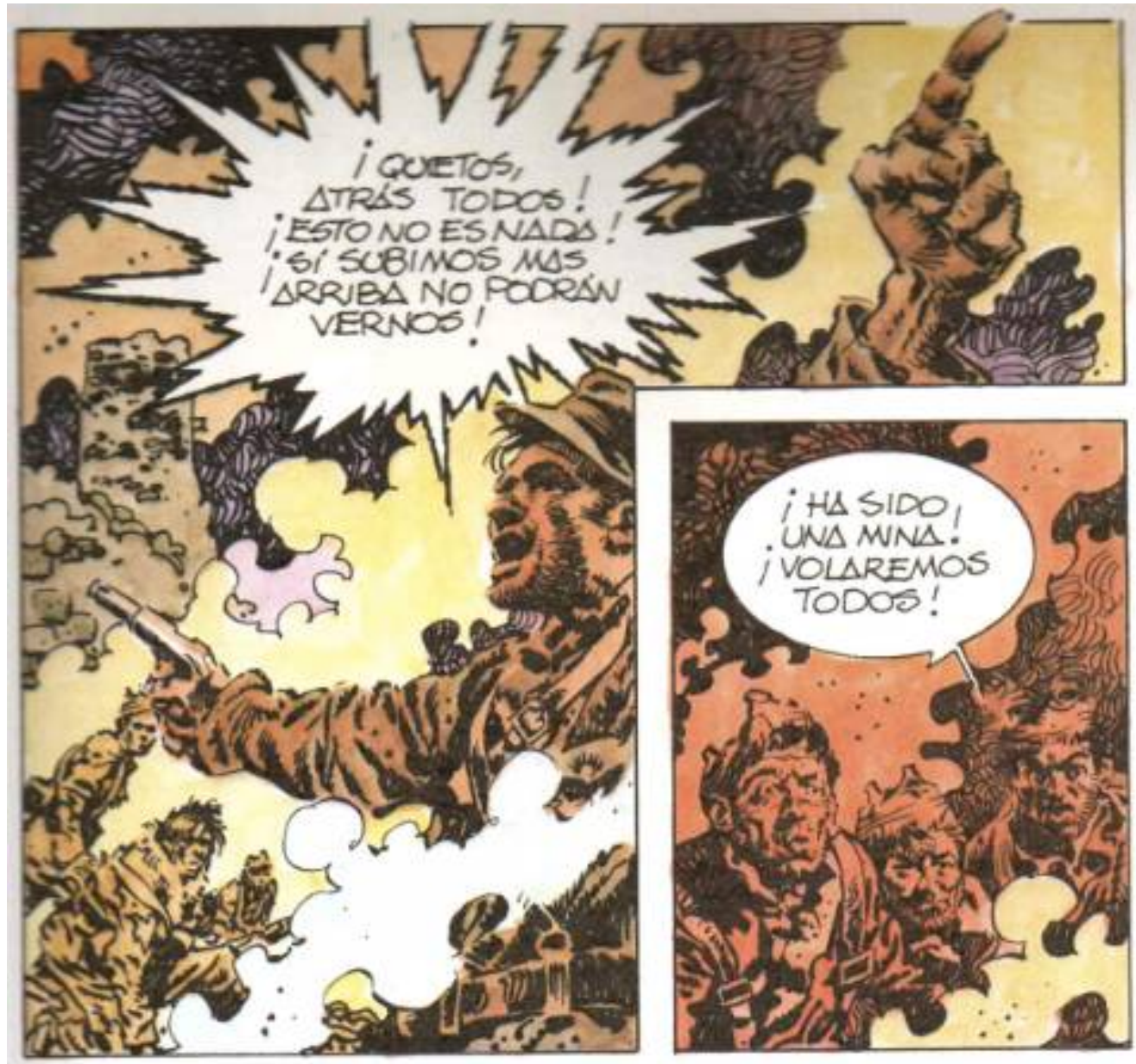

Illustration 1 : AntonioHernández Palacios, Río Manzanares, Vitoria, Ikusager, 1979, p. 21, cases 1-2-3. 

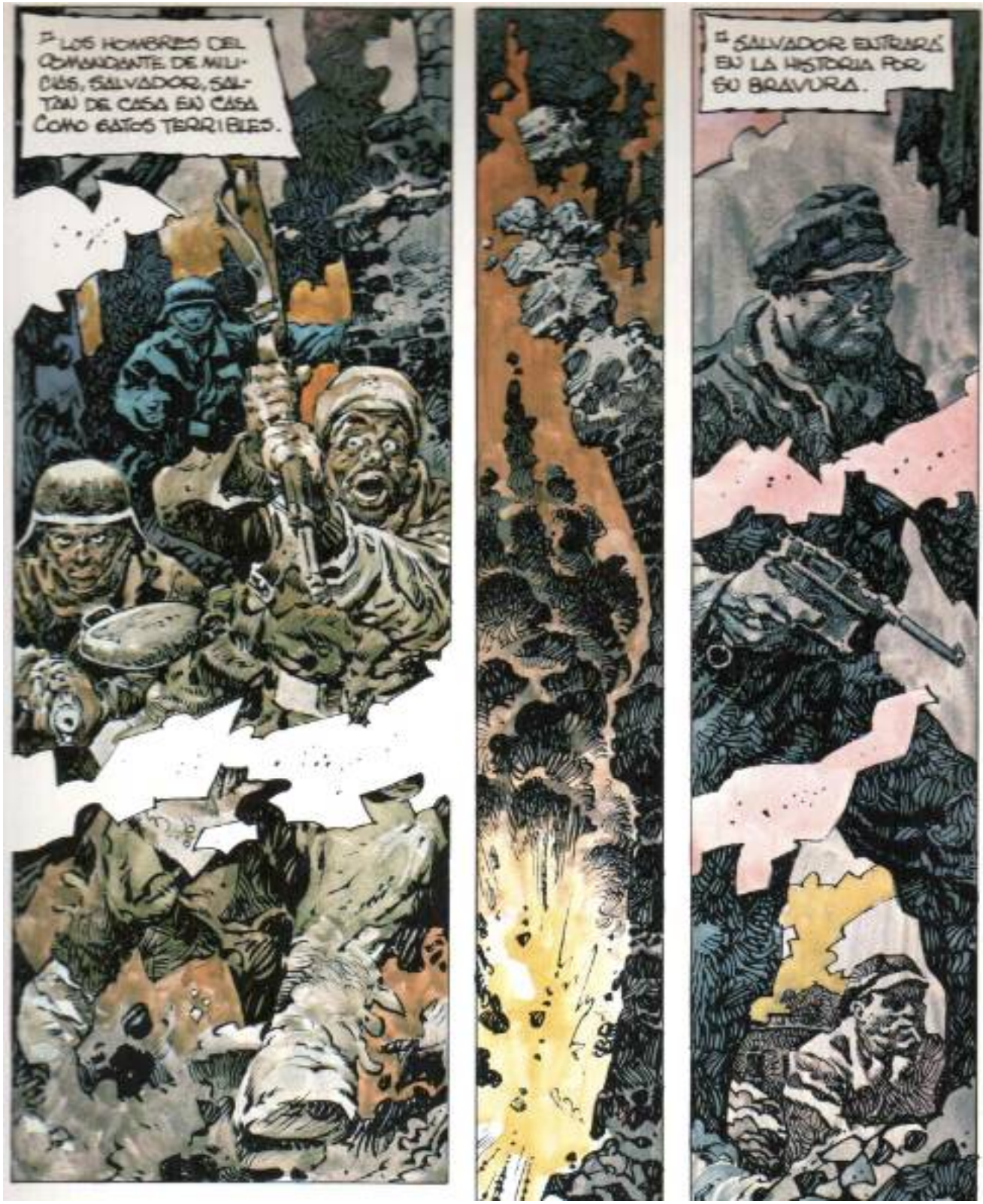

Illustration 2 : AntonioHernández Palacios, Eloy, uno entre muchos, Vitoria, Ikusager, 1979, p. 17, cases 1-2. 


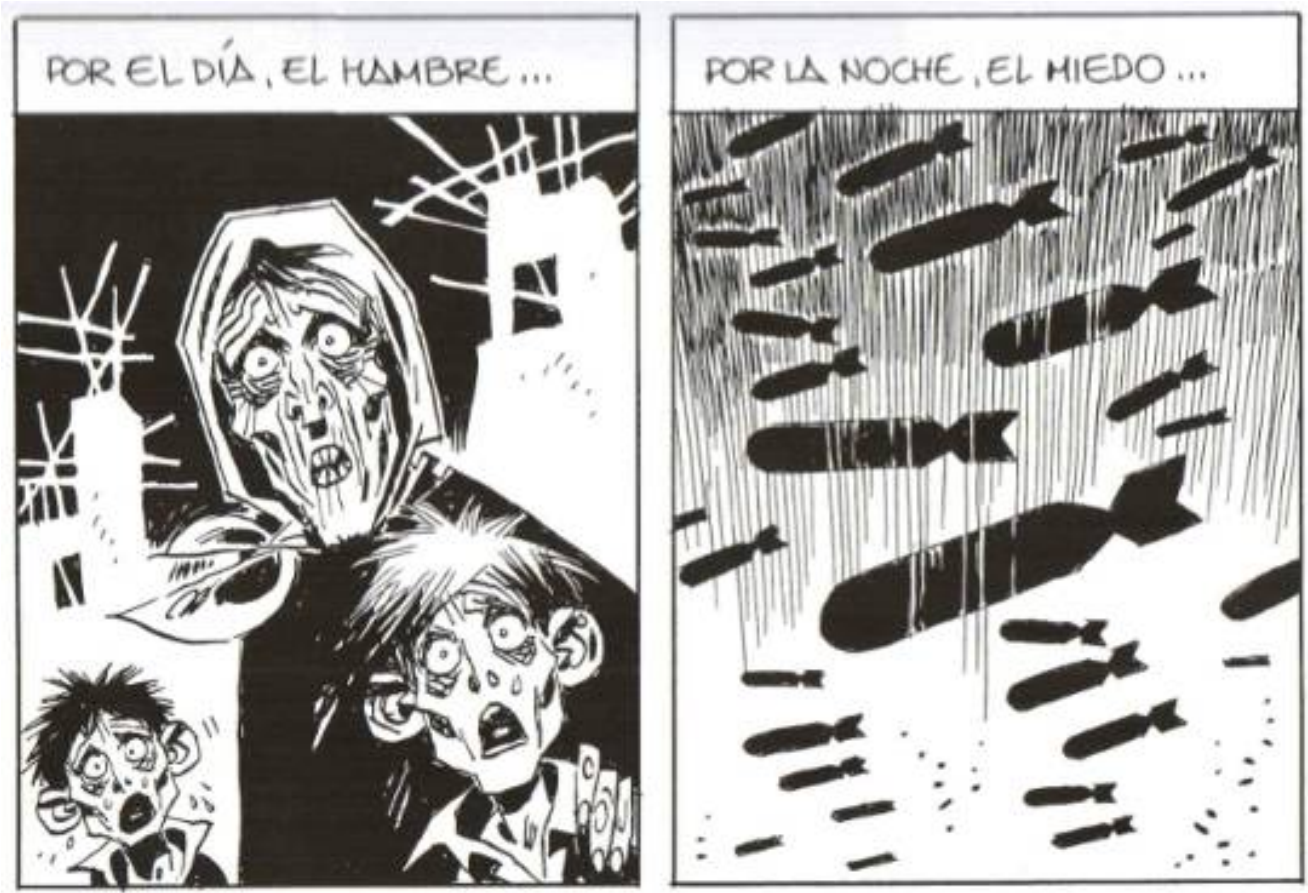

Illustration 3 : Carlos Giménez, 36-39. Malos Tiempos, vol.3, Barcelone, Glénat, 2008, p.7, cases 2-3. 


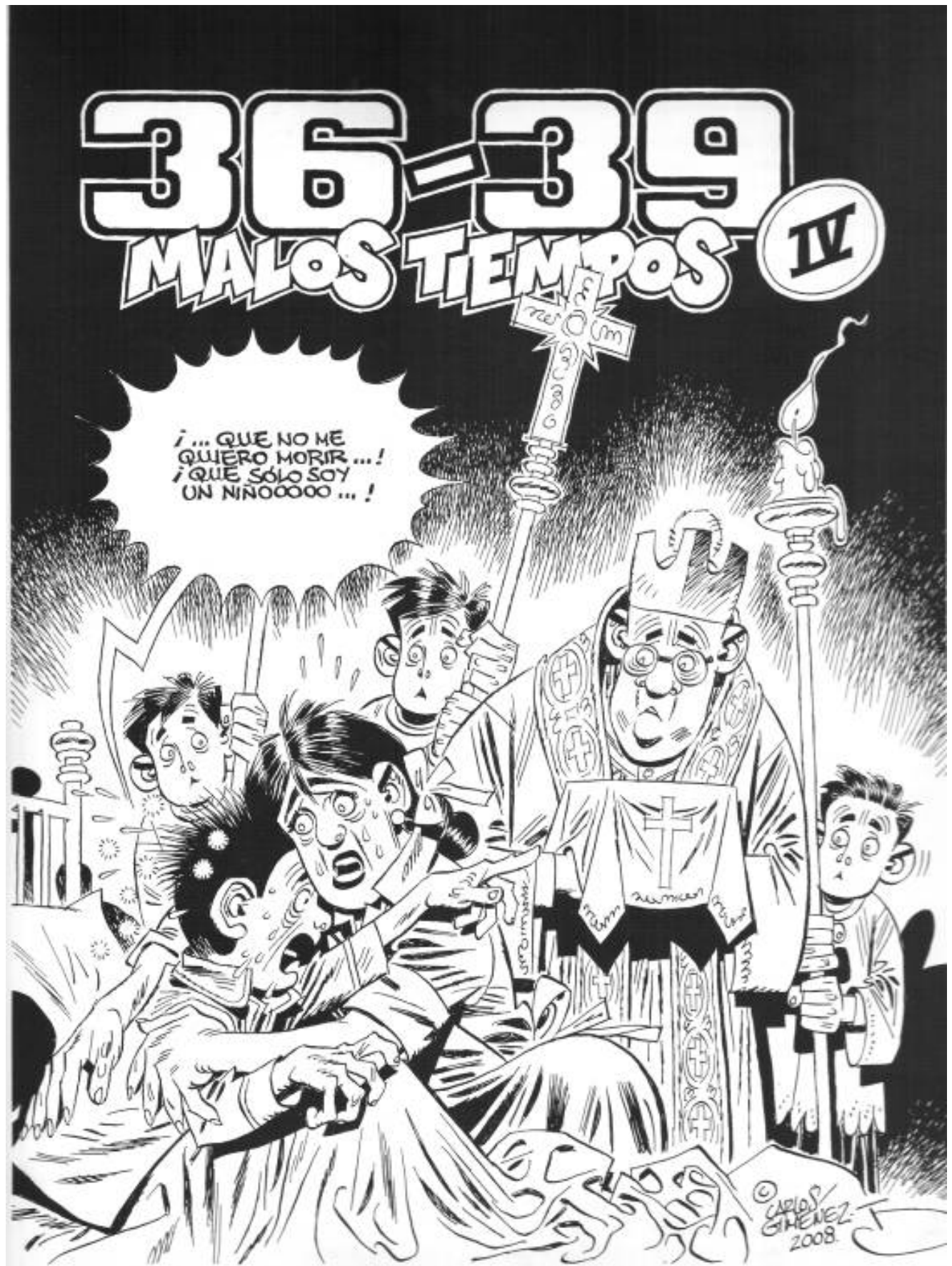

Illustration 4 : Carlos Giménez, 36-39. Malos Tiempos, vol.4, Barcelone, Glénat, 2008 (illustration de couverture).

\section{Notes}

1. Cf. Maryse Bertrand de Muñoz, «La guerre civile espagnole et la production romanesque des quinze dernières années face à celle de la transition à la démocratie », dans Viviane Alary et Danielle Corrado, La guerre d'Espagne en héritage. Entre mémoire et oubli (de 1975 à nos jours), Clermont-Ferrand, Presses Universitaires Blaise Pascal, 2007, p. 31-46.

2. Michel Porret, Objectif bulles, Genève, Georg, 2009, p. 12.

3 . Jérôme Delaplanche et Axel Sanson, Peindre la guerre, Paris, Éditions Nicolas Chaudun, 2009, p. 138. 4. S.v. «guerrier» dans Le grand Robert.

5 . Conflit international, lointain et surtout victorieux qui se prête fort bien à la glorification et à l'héroïsation du peuple espagnol. 
6. Pour Paul Ricoeur, l'origine et l'identité narrative de bien des «communautés historiques» sont structurées autour d'événements marquants qui sont souvent des faits guerriers. Cf. Temps et récit, t. 3 , Paris, Le Seuil, 1985, p. 339.

7 . Face, semble-t-il, à la complexité du sujet (le risque de censure était très élevé), d'autres guerres, plus lointaines et glorieuses, ont en revanche été le théâtre d'action de nombreux personnages dont quelquesuns ont connu un succès retentissant (pensons à El Capitán Trueno et à El Cachorro qui se battaient en plein Moyen Âge, à El Jabato, jeune Ibère rebelle à l'envahisseur romain ou encore à El guerrero del Antifaz dont on trouvera une analyse détaillée dans l'article de Pierre Paul Gregorio).

8 . Citons, par exemple, Las serpientes ciegas (BDbanda, 2008) de F. Hernández Cava et B. Seguí (Premio Nacional del Cómic 2009) ou encore El Arte de volar de A. Altarriba et Kim (Premio Nacional del Cómic 2010 et Prix du meilleur album 2010 du Salon de la BD de Barcelone).

9 . Précisons que l'implication de Glénat dans ce domaine n'est due qu'à la présence et la volonté d'un seul homme, Antonio Martín, authentique figure du monde de l'édition BD en Espagne.

10. À notre connaissance, Palacios est le seul auteur de BD à avoir participé à la Guerre civile et à avoir pu (quarante ans après la fin du conflit et seulement quatre ans après la mort de Franco) retranscrire graphiquement cet événement. Peut-être plus exceptionnel encore, Palacios, en dépit de son jeune âge, a connu la guerre des deux côtés : engagé d'abord auprès des républicains (son bord politique), il finira enrôlé de force dans la «División Azul» et partira sur le front russe aux côtés des troupes franquistes. Cf. le prologue de Palacios dans Eloy, uno entre muchos et la nécrologie de F. H. Cava intitulée «Un dibujante sin pausa » (El Mundo, 31 janvier 2000).

11 . Ernesto Santolaya décrit avec justesse l'œuvre de Palacios en la caractérisant de «comprometida pero sin partidismos » (engagée mais non partisane), dans « Antonio Hernández Palacios, dibujante », El País, $1^{\text {er }}$ février 2000 (section « Necrológicas »).

12 . Eloy pour Eloy, uno entre muchos et Río Manzanares ou Gorka pour 1936. Euskadi en llamas et Gorka Gudari. On retrouvera les notices bibliographiques en fin d'article.

13. Carlos Giménez, Paracuellos, Barcelone, Glénat, 2000. À noter que Paracuellos (Fluide Glacial, 2009) a reçu à l'occasion du $37^{\mathrm{e}}$ festival de la bande dessinée d'Angoulême en 2010 le «Prix du Patrimoine ».

14. Carlos Giménez, 36-39. Malos Tiempos, vol. 1-2-3-4, Barcelone, Glénat, 2007-2009.

15 . Concept employé par Thierry Groensteen dans son Système de la bande dessinée, Paris, PUF, 1999.

16. Voir note $\mathrm{n}^{\circ} 7$.

\section{Pour citer cet article}

\section{Référence électronique}

Benoît Mitaine, « Une guerre sans héros ? La Guerre civile dans la bande dessinée espagnole (1977-2009) », Cahiers de la Méditerranée [En ligne], 83 | 2011, mis en ligne le 15 juin 2012, consulté le 10 janvier 2015. URL : http://cdlm.revues.org/6256

\section{Référence papier}

Benoît Mitaine, «Une guerre sans héros ? La Guerre civile dans la bande dessinée espagnole (1977-2009) », Cahiers de la Méditerranée, 83। 2011, 227-236.

\section{À propos de l'auteur}

\section{Benoît Mitaine}

Maître de Conférences au Département d'Espagnol de l'Université de Bourgogne, il est l'auteur de divers articles sur la bande dessinée, l'image fixe et le roman espagnols contemporains. Il a co-édité Lignes de front. Guerre et totalitarisme dans la bande dessinée (Georg, 2011) et est l'un des coauteurs du Lexique bilingue des arts visuels (Ophrys, 2011). Il co-dirige actuellement un programme de journées d'études sur le sujet « Bande dessinée et adaptations. Du texte aux images / De la page à l'écran».

\section{Droits d'auteur}

(C) Tous droits réservés 


\section{Résumés}

La bande dessinée espagnole de l'après-franquisme, à contre-courant de tous les autres secteurs de l'édition depuis 20 ans, n'a jamais témoigné d'un grand intérêt pour le sujet de la Guerre civile qui a déchiré l'Espagne entre 1936 et 1939. Autre sujet d'étonnement, les représentations qu'elle a pu en faire s'opposent radicalement au schéma classique et attendu de la bande dessinée d'aventure qui exalte la figure du héros de guerre. Les œuvres étudiées dans cet article (la tétralogie de Carlos Giménez et celle de Antonio Hernández Palacios), aussi différentes soient-elles, ne montrent qu'une chose : la tragédie de la guerre.

Contrary to all other publishing sectors over the past two decades, Spanish comics and graphic novels of the post Franco years have never demonstrated much interest in dealing with the Civil War which tore Spain apart between 1936 and 1939. Also surprising, the representations they offer contrast radically with the classic conventional forms of adventure comics exalting war hero figures. Despite their differences, the works studied in the article, Carlos Giménez's and Antonio Hernández Palacios's four-volume opuses, depict but one thing, the war's tragedy.

\section{Entrées d'index}

Mots-clés : Antonio Hernández Palacios, bande-dessinée espagnole, Carlos Giménez, Guerre civile espagnole

Keywords : Antonio Hernández Palacios, Carlos Giménez, Spanish Civil War, Spanish comics 